\title{
Programmable Hall Effect Sensing Element
} - for Camshaft \& Crankshaft Position Sensors -

\section{Olivier Marle $^{1)}$ Jae-Bong Koo $^{2)}$}

\author{
1) CONTINENTAL, Chassis \& Safety Division, Passive Safety \& Sensorics Business Unit \\ 1 Avenue Paul Ourliac, 31036 Toulouse Cedex, France (E-mail: olivier.marle@continental-corporation.com) \\ 2) CONTINENTAL, Chassis \& Safety Division, Passive Safety \& Sensorics Business Unit. \\ 1 Avenue Paul Ourliac, 31036 Toulouse Cedex, France (E-mail: jae-bong.koo@continental-corporation.com)
}

Received on June 30, 2016

Presented at the JSAE Annual Congress on May 27, 2016

\begin{abstract}
The programmable Hall Effect Sensing Element developed by Continental allows measuring camshaft and crankshaft positions precisely. This is essential for modern cars designing clean vehicles with increased engine power efficiency and minimized fuel consumption. The high flexibility of the sensing element programming allows adapting the sensor to all customer requirements and various engine environments. The sensing element is particularly robust against temperature variations during stop-start and target wheel vibrations at power-up by preventing any wrong calibration then. The choice of the application and programming of associated parameters is done at the End-of-Line thanks to a dedicated communication protocol and an embedded EEPROM.
\end{abstract}

KEY WORDS: heat engine, camshaft \& crankshaft sensors, stop-start, variable valve timing, combustion engines, gearbox sensors Electric Fields [A1]

\section{INTRODUCTION}

One of the major challenges of thermal engines of modern cars is the design of clean vehicles with increased power efficiency and minimized fuel consumption. This challenge cannot be achieved without a fully optimized engine control management. Camshaft and Crankshaft position sensors are two essential electronic components which allow determining the position of the engine pistons and valves precisely (Figure 1).

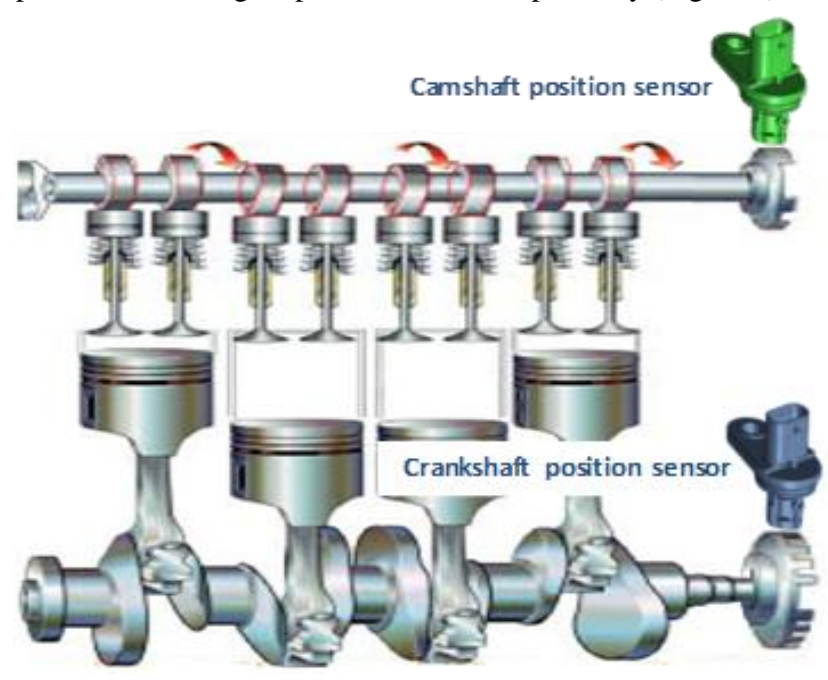

Fig. 1 Sensors location in engine

Due to the continuous increased number of electronic components and embedded systems, another major challenge of modern cars is to remain price competitive. Thus, in order to comply both technical and economic objectives, we developed a sensor concept based on one fully programmable Hall Effect sensing element. The sensor can be used for TPO twist insensitive camshaft applications, regular crankshaft applications and bi-directional crankshaft applications for stop-start systems. It can be used for gearbox applications as well.

The sensor has been especially designed to operate with multiple gear wheels, having a wide variety of Tooth-Notch mechanical dimensions, and it was optimized to operate in multiple conditions of temperatures, air-gap, target wheel runout and mechanical stress. It integrates self-adaption mechanisms allowing high signal phase accuracy down to $0.5^{\circ}$ and high signal repeatability better than $0.015^{\circ}$ (three standard deviations).

\section{STATE OF THE ART}

Crankshaft and Camshaft position sensors are most of the time active contactless sensors which need to be powered in order to provide a signal. These are magnetic sensors which integrate either a Hall Effect Integrated Circuit (IC) ${ }^{\text {(1) (2) (3) }}$ or a magneto-resistive IC using anisotropic magneto resistance $(\mathrm{AMR}){ }^{(4)}$ or giant magneto-resistance $(\mathrm{GMR}){ }^{(5)}$. Aside from those, less expensive passive sensors based on inductive technology exist. These were largely used formerly but are limited in terms of functionality.

The active sensors measure and transform the magnetic field induction modulated by the passing of the teeth and notches of a ferromagnetic cogwheel (also called target wheel - Figure 2) which is fixed to the camshaft or the crankshaft. They provide a two levels electrical signal (generally $0-5$ Volts) which represents the image of the teeth and notches of the target wheel 
and which is interpreted by the engine controller. For a transmission speed sensor, the modulation of the magnetic field induction can directly be conducted by the gears of the transmission. Magnetic field induction is produced by a permanent magnet located either at the back side of the sensor sensing element or around it. Generally, magnets for crankshaft and camshaft position sensors are different which consequently implies the design of different sensors.

Magnetic field induction can also be produced by the target wheel itself. This is based on the prerequisite that it comprises a part loaded magnetically which integrates magnetic tracks providing alternating South and North poles. Figure 2 also shows the example of a metallic plate that integrates a magnetic rubber vulcanized on the base plate.

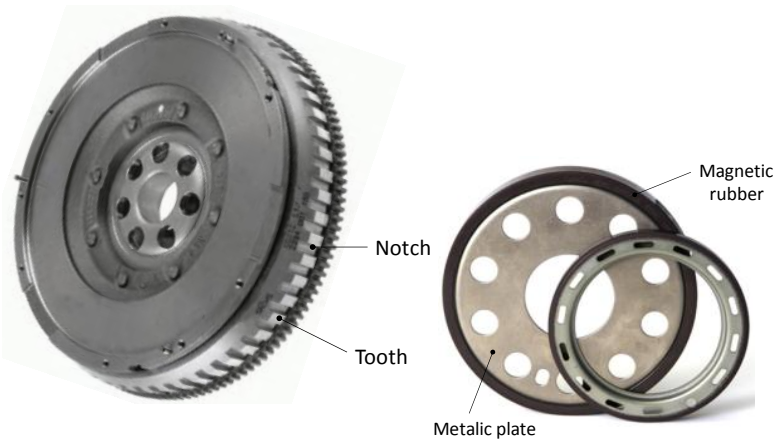

Fig. 2 Target wheels

60-2 teeth ferromagnetic crank target wheel- Source: LUK Magnetic encoders - Source: Hutchinson

Other technologies of measurement also exist as eddy current sensors but they are less common. Many suppliers of sensing elements exist on the market and propose standard IC solutions in order to allow crankshaft and camshaft position measurement or to measure the velocity of transmission gear wheels.

Even though the crankshaft, camshaft position sensors and transmission speed sensors are incremental using the same base technologies of measurement (Hall effect or magneto-resistance), every single one has functional requirements that often differ from each other.

Consequently, these significant differences determine the sensing elements' architectures and the signal processing algorithms. Not to mention the diversity of the target wheels due to an increasing number of car manufacturers and to a lack of design rules concerning some specific dimensions of the target wheels. Indeed, those are generally optimized to fit mechanical and process constraints. However, they sometimes do not comply with the essential magnetic requirements for an optimum operation of the sensor and a simplification of the sensing architectures.

\subsection{Crankshaft position sensor}

One of the main goals of a crankshaft position sensor is to produce highly repeatable signal edges in regards to the corresponding mechanical edges of the target wheel. This repeatability (also called phase jitter) valid for any ambient temperature, distance of the sensor to the target wheel, target wheel rotation speed or any other parameter of environment, is required to detect engine miss fire events which affect the engine performance and environmental pollution. One essential requirement is to achieve a sensor signal repeatability down to one hundredth of degrees. The signal repeatability is linked to several parameters such as the technology of the sensing element, the electronic noise, the magnetic circuit, the target wheel ToothNotch pattern, the temperature, the air-gap etc. Figure 3 shows the magnetic edges where the sensor switches. The steeper the magnetic edges are, the lower the sensor exhibits signal phase jitter.

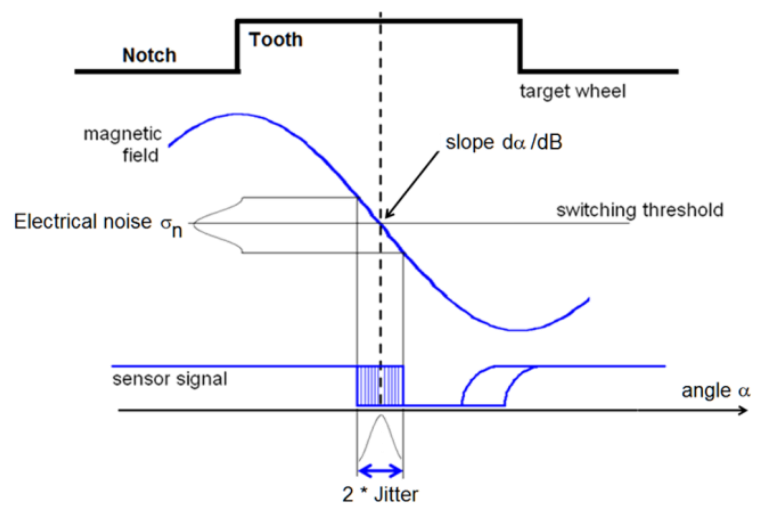

Fig. 3 Sensor signal phase jitter

The hereafter formula provides the basic relationship between phase jitter $\left[{ }^{\circ}\right]$, electronic noise $\sigma_{\mathrm{n}}$ [Gauss], magnetic slope $\mathrm{d} \alpha / \mathrm{dB}[\mathrm{mm} / \mathrm{Gauss}]$ and target wheel radius $\mathrm{R}[\mathrm{mm}]$.

$$
\operatorname{Jitter}\left[{ }^{\circ}\right]=\frac{\sigma_{n}[\mathrm{G}] * d \alpha / \mathrm{d} B[\mathrm{~mm} / \mathrm{G}] * 360^{\circ}}{2 * \pi^{*} R[\mathrm{~mm}]}
$$

The absolute position accuracy required for the electrical edges compared to the corresponding mechanical edges is requested to be lower than $0.5^{\circ}(0.14 \%)$ without considering the phase variation over speed which is generally compensated by the engine controller. Depending on the system, the sensor signal edges can occur either at the location of the mechanical edges of the target wheel or in the middle of the teeth and notches (Figure 4). This consequently leads to different architectures of measurements and hence to the application of different sensors. To meet given requirements of signal repeatability and accuracy, the crankshaft position sensors generally make use of a differential measurement architecture which requires the sensing elements to align accurately in regard to the target wheel. 
This particularly applies for bi-directional crankshaft position sensors which integrate two channels of measurement of the magnetic field induction in order to determine the direction of rotation of the target wheel in addition. This direction of rotation is coded in the sensor signal in the form of a programmable pulse width (Figure 4). It is used by the engine controllers which integrate a stop-start strategy to continuously monitor the position of the engine and thus to avoid a full engine resynchronization at restart after a vehicle stop phase. Bidirectional crank sensors integrate special signal processing algorithms in order to avoid wrong pulses during engine stop phases and wrong signal information at restart.

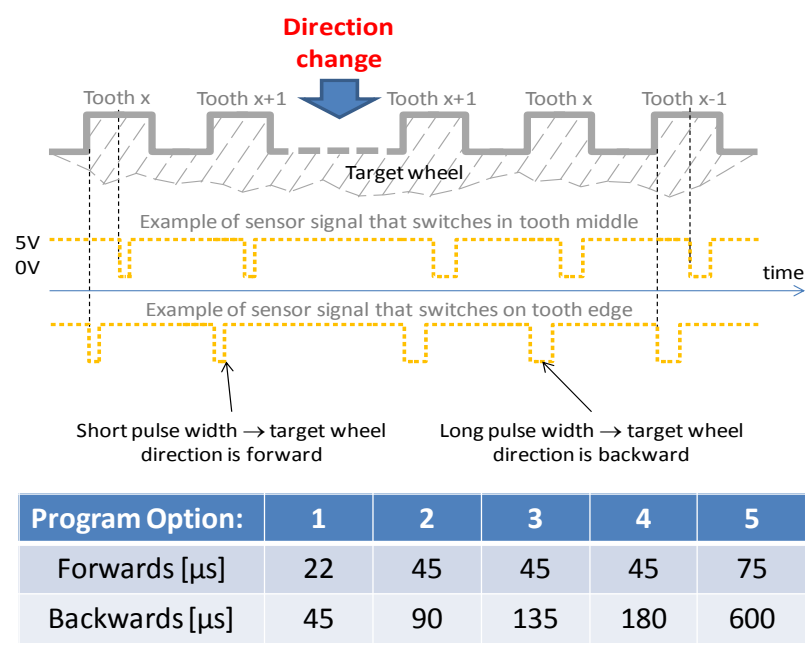

Fig. 4 Bi-directional crankshaft position sensor with programmable signal pulse widths

\subsection{Camshaft Position Sensor}

Unlike crankshaft position sensors, camshaft position sensors do neither require a highly repeatable signal nor measure the target wheel motion direction. But, they do request other features such as Twist Insensitivity or True Power ON.

\subsubsection{Twist Insensitivity}

"Twist Insensitivity" means the obligation of the sensor to constantly provide a correct signal at power-up even when turning the sensor around its own axis of $360^{\circ}$ (Figure 5). Thus, such sensors require a measurement cell architecture which is insensitive to the direction of rotation of the target wheel. This means that differential measurement architecture of previously mentioned crankshaft position sensors cannot apply. These sensors are generally based on a planar Hall Effect measurement principle using only one cell which is located at the center of the sensor. When the sensor is turned around its axis, the magnetic field induction (produced by the permanent magnet of the sensor) remains unchanged and the sensor signal response towards the target wheel stays identical (within the sensing element positioning tolerance inside the sensor).

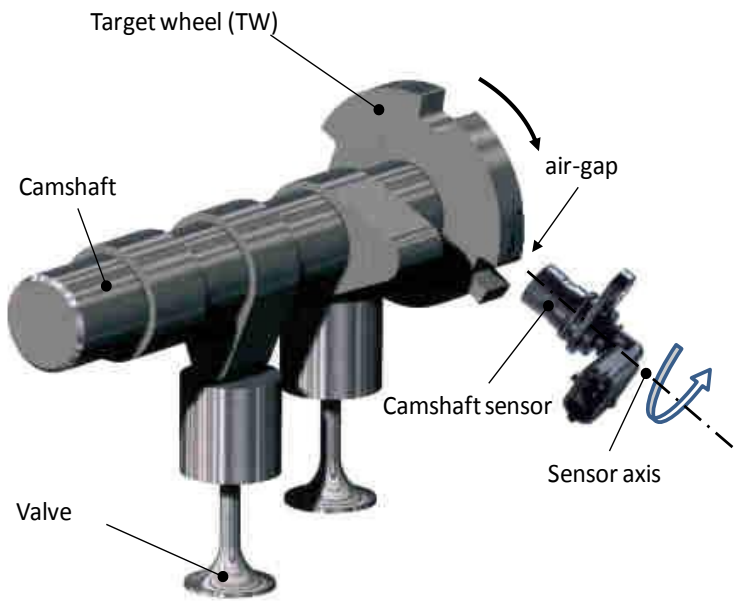

Fig. 5 Twist insensitivity

This functionality offers car manufacturers the enormous advantage of using the same sensor on various engine blocks knowing that several sensors must be mounted within one block (e.g. V shape engine). It is made possible by the fact that camshaft position sensors don't need as much accuracy as crankshaft position sensors. Here the mono cell architecture of measurement is rather well adapted. Generally, the required signal phase accuracy of camshaft position sensors complies with the degree range set by the Variable Valve Timing (VVT) requirement. The engine synchronization requests less CAM signal phase accuracy.

Nevertheless, certain car manufacturers seek to increase the accuracy of camshaft position sensors and intend to have it on a similar level as of the one of crankshaft position sensors. This request is particularly challenging as the accuracy of the sensor signal electrical edges strongly depends on the diameter of the camshaft target wheel which is about one to eight times smaller than the diameter of crankshaft target wheels. The smaller the diameter of the target wheel, the more degraded the angular accuracy of the sensor-target system. That goes without mentioning the influence of target wheel run out (air-gap variation due to target wheel assembly on camshaft and manufacturing tolerances) which also degrades the accuracy in case of a mono cell architecture of measurement, even by using sophisticated techniques of signal processing to minimize the run out effects.

Consequently, a differential architecture of measurement similar to the one used in crankshaft position sensors is better adapted. Indeed, it permits to have a signal switching point (also called threshold), practically independent of the air-gap variations and thus of the target wheel run out. Certain car 
manufacturers use sensors integrating differential architecture of measurement but these sensors are twist sensitive. Just like crankshaft position sensors, the sensor signal electrical edges can be generated either at the location of the target wheel mechanical edges or in the middle of the teeth and notches. This leads to different architectures of measurement and as a result to different sensors.

\subsubsection{True Power ON (TPO)}

An additional requirement of certain camshaft position sensors is the TPO function. This function allows the sensor to indicate the engine controller, if it is in front of a tooth or a notch, as soon as it is powered and under various conditions of air-gap, temperature and sensor assembly towards the target wheel. This requirement does not apply for crankshaft position sensors. To do this, the sensor provides the logical state "high" over a tooth and the logical state "low" over a notch (or the opposite). Consequently, the synchronization of the engine at start-up can be done more quickly thanks to the angular coding provided by the target wheel. The TPO function is generally implemented on twist insensitive sensors. It requires a 100 percent calibration of the sensing element-permanent magnet system due to the assembly and components tolerances. To allow a nearly temperature independent operation, it is required to compensate the sensing element by adapting it to the magnetic circuit. Moreover, a special magnetic circuit is needed in order to produce a mean static field induction value at the sensor switching point close to zero Gauss (Figure 6).

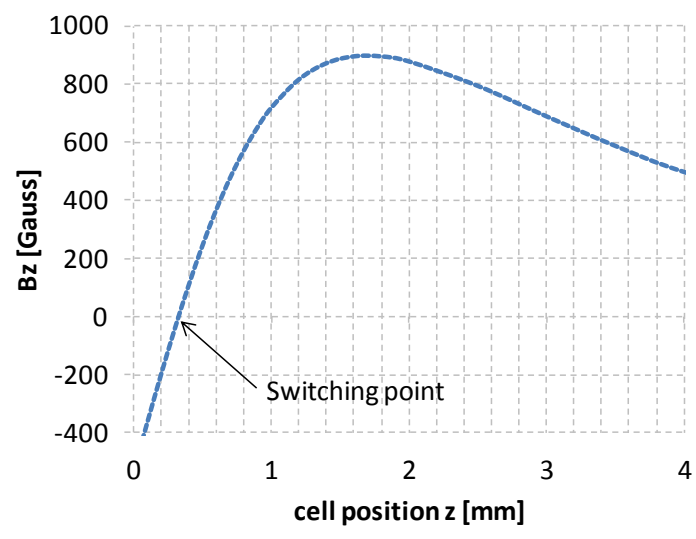

Fig. 6 Sensor switching point close to zero Gauss

This allows minimizing the drifts of magnetic offsets over the temperature and thus reducing the losses of sensor sensitivity and signal phase variation over the temperature. Moreover, it is possible to make sensors capable of operating on relatively high air-gap ranges (up to $2.5 \mathrm{~mm}$ or more), until the Tooth-Notch mechanical pattern of the target wheel allows it.
Generally, TPO function does not guarantee high sensor signal phase accuracy at start-up as the point to switch the sensor signal is fixed and thus causes phase accuracy variations depending on the air-gap and the target wheel diameter. Variation deviates in general by a few degrees.

To guarantee higher signal phase accuracy needed mainly for VVT function (see also limp home function), self-learning algorithms have to be used.

Most of the previously mentioned concerns can be solved by the use of a magnetic encoder. However, this method is more difficult to handle and currently more expensive compared to existing ferromagnetic target wheels (e.g. use of the camshaft pulley as camshaft target wheel or the camshaft itself).

\section{PROGRAMMABLE HALL EFFECT SENSING ELEMENT}

As mentioned in the previous chapter, the camshaft, crankshaft position sensors and speed sensors for gearbox applications integrate a wide range of requirements. All of them involve very different sensing elements architectures and technologies, signal processing algorithms or methodologies as well as magnetic circuits. In addition, there is no existing standard packaging of the sensing elements which thus avoids a mechanical standardization of the complete sensor module.

It works without considering certain problems linked to target wheels which sometimes require specific magnetic signal processings to allow the sensor working properly. Examples are magnetic inhomogeneities due to mechanical imperfections (e.g. burrs or strikes), not well adapted Tooth-Notch mechanical patterns (notches too short), magnetization process (pole wheels) or high level manufacturing tolerances. Therefore, sensing elements' suppliers set up solutions that give answers to particular problems in regards to function efficiency.

If one takes a closer look at the global level in line with previously mentioned sensors, all these ones are incremental sensors mainly aiming to provide an electric signal that comprises two levels (voltage or current). Their transitions shall be perfectly phased to the corresponding mechanical transitions produced by the passing of the teeth and notches (or magnetic poles) of the target wheels. This electrical image must be independent of all environmental conditions of the sensor and must be available from the sensor power-up. To cover all the application's requirements, several sensing elements of the market are needed.

Currently, the sensing element represents more than 50 percent of the complete sensor module value. Therefore, electrical functions programmability and sensing module design 
standardization are mandatory to remain competitive, knowing these commodity products only offer limited rooms for innovation.

\subsection{Integrated flexible sensing architecture}

The goal was to create a programmable magneto-electrical module capable to operate with all the applications (Figure 7). The module was designed in a way that it offers a unique shape which can be assembled or molded in a plastic material in order to create the OEM's specified outline dimensions, mounting bracket and connector. The magneto-electrical module insures a perfect cohesion between sensing element and magnet which is essential to guarantee a high-level design stability in very harsh conditions of environment and durability. The module includes a standard connections area to attach a conductive lead frame fulfilling the OEM's requirements. The dimensions of the module have been defined to fit overall known sensor dimensions.

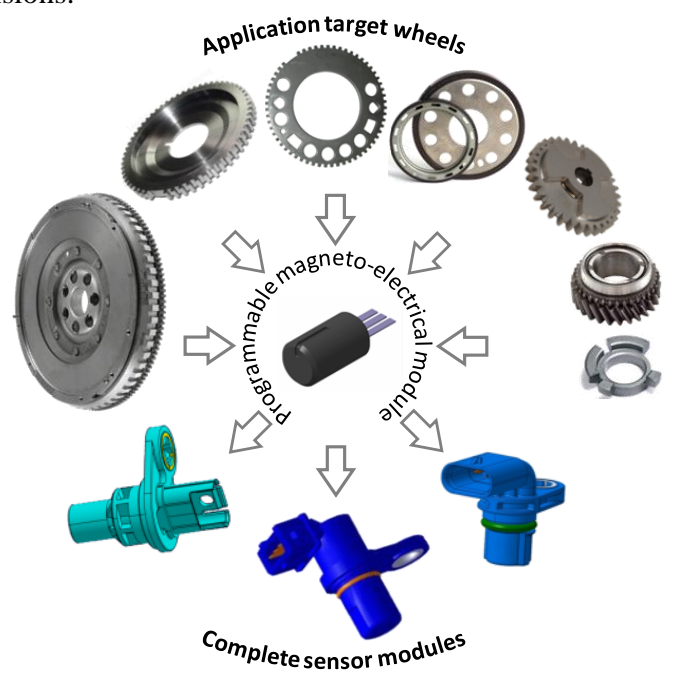

Fig. 7 Programmable magneto-electrical module

One of the most important innovations was to integrate all the functions related to camshaft, crankshaft and transmission speed applications in one unique sensing element. This represents the first IC for those automotive applications with such a technology.

From the measurement point of view, the device integrates a flexible sensing architecture allowing selecting the appropriate sensing elements depending on the application. Several signal processing algorithms can be selected from digital point of view to reach the desired functional requirements. The selection of the parameters and functions is performed via a four times 22 bits embedded EEPPROM. Programming is done after the sensor assembly has been completed. This permits compensating all errors due to the sensor manufacturing process and components tolerances. In addition, it allows to activate special features or to vary parameters in order to adapt the sensor to target wheel imperfections or specific application requirements.

The device permits to be used as a mono cell sensing element for TPO twist insensitive camshaft position sensor or a multicells sensing element for twist sensitive camshaft position sensor, regular and bi-directional crankshaft position and transmission speed sensors. Particular care has been taken to prevent the device performing any false signal calibration at power-up due to unexpected events and to always quickly recover to full functionality.

\subsection{Specification and measurement results}

For bi-directional crankshaft position sensors, digital signal processing has been especially optimized to track all forward and backward movements of a large number of target wheels in various environmental conditions. In addition, it has been designed to guarantee an error-free system at engine restart and during stop phases when some large temperature and air-gap changes may occur. Several signal pulse width protocols can be selected to indicate the cogwheels motion direction (Figure 4).

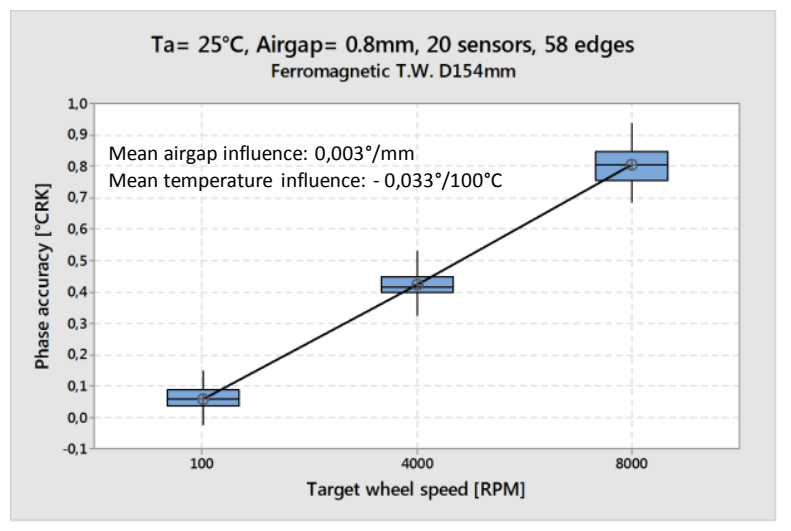

Fig. 8 Crankshaft position sensor accuracy

In the end, the algorithms guarantee high signal phase accuracy better than $0.5^{\circ}$ speed effect excluded and a low signal phase jitter (see quartile Figures 8 and 9).

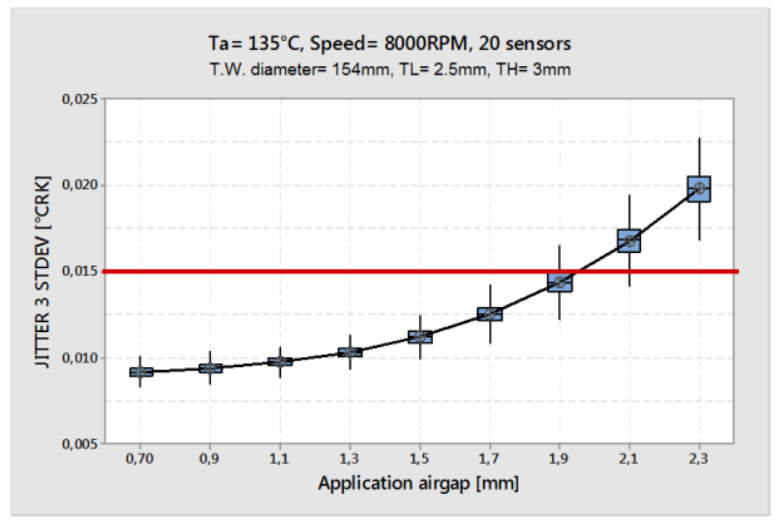

Fig. 9 Crankshaft position sensor signal jitter 
For TPO twist insensitive camshaft position sensor, the algorithms permit the sensor providing the full accuracy after a few edges following the sensor power-up and minimizing the effects of the target wheel run-out (Figure 10). The device is fully functional even with a large run-out of one mm. It allows adapting the signal switching position versus cogwheel edges in order to fit specific OEM's signal phasing requirements.

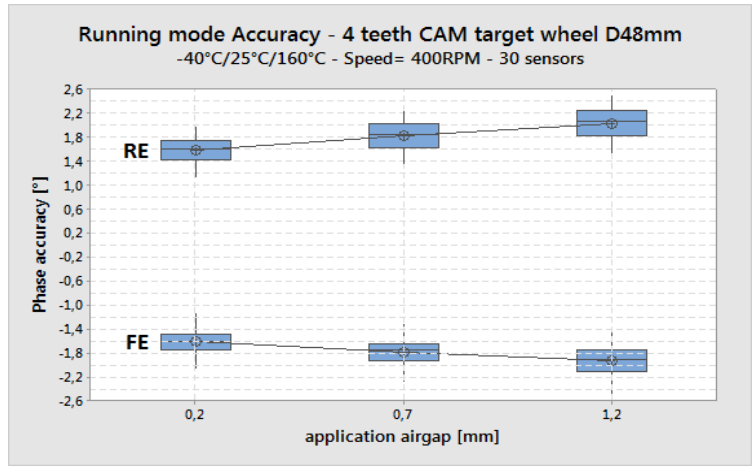

Fig. 10 Mono-cell camshaft sensor phase accuracy

Finally, the device can also operate in transmission applications requesting to be robust to air-gap jumps of $\pm 0.5 \mathrm{~mm}$ and to be immune to target wheel vibrations when the vehicle is being stopped. For direction information sensor type, several signal pulse width protocols can be programmed also including some standstill pulse protocols for diagnosis purpose.

To allow high performances in terms of accuracy and air-gap over temperature, the sensing elements signal paths have been fully optimized. They integrate a full chopper-stabilized (6) system and use a special technique to compensate the sensitivity variations due to mechanical stress ${ }^{(7)}$. This is essential for overmolding the IC using a thermoplastic injection molding process or using the IC in harsh conditions. Particular care has been taken for minimizing the electrical noise to provide the best jitter performances that are also valid for sensing of small diameter camshaft target wheels or by using low remanence magnets.

The device has a wide magnetic field induction working range capability offering the possibility to operate with various magnetic materials (rare earth or low remanence materials). The choice of the material is done by programming. For magnetic pole wheels, the device has no need of a magnet.

To validate and fully optimize the device algorithms and digital features, a complete Matlab ${ }^{\circledR}$ model has been created. This model operates with real analog field induction measurements of the application target wheels, thus allowing to simulate real cases and to correlate the results with physical samples. Furthermore, the Matlab $^{\circledR}$ calculations permit to consider systematic or random errors that are difficult to produce on a cogwheel test bench system in order to verify the algorithms behavior and to test their limits. In addition to the simulations and laboratory characterizations, the sensing element has been fully validated through various environmental testing conditions at IC level and on complete sensor module level.

\section{CONCLUSION}

The technologies for camshaft, crankshaft position and transmission speed sensors as well as integration activities of the required functions in a unified sensor element have been presented in this article.

The development of a programmable magneto-electrical module based on a unified sensing element enabled the implementation of standard design rules for the complete sensor module in order to ease the manufacturing processes and to allow the accumulation of production volumes. Therefore it offers a cost efficient solution to adapt to the market needs in terms of competitiveness regarding technology and price.

Moreover, this technical solution permits to shorten development times thanks to the possibility of re-using the same mechanical sensor base for several applications without performing a full revalidation. It is also possible to optimize validations because of subsidiary qualification activities and to enhance reliability due to associated qualification results from diverse applications as well as to reach a higher quality level because of more focused process controls.

Today, the integration of the overall functions, e.g. TPO and twist insensitivity for camshaft sensor and high repeatability for crankshaft sensor, in a unified sensing element are important cost factors. Therefore, a next innovation step has been projected in order to remain competitive and to easily react on a further tightening of requirements in terms of performance and reliability. This will consider further integration measures, including new sensing element technology and packaging improvements as well as enhanced functionalities in terms of digital signal processing and electrical performances.

\section{REFERENCES}

(1) Allegro Microsystems LLC, datasheets of True Power ON Camshaft sensor IC's ATS675LSE, www.allegromicro.com, (Access Date 06/21/2013) and ATS601LSG (Access Date 12/31/2013)

(2) Infineon Technology AG, Product briefs of Programmable True Power ON Camshaft sensor IC's TLE4983C, www.infineon.com, (Access Date 11/2011) and TLE4984C (Access Date 03/2012)

(3) Allegro Microsystems LLC, Product information of Crankshaft sensor IC's ATS658 and ATS694 for stop/start systems, www.allegromicro.com, (Access Date 01/04/2012)

(4) NXP Semiconductors, datasheets of Integrated rotational speed sensor IC's KMI16-18, www.nxp.com, (Access Date 09/05/2000)

(5) Infineon Technology AG, TLE5027 differential i-GMR speed sensor IC with direction detection, product brief, www.infineon.com, (Access Date $07 / 03 / 2013$ )

(6) Scientific Bulletin, Series C, Vol. 73, Iss. 4 (2011) http://www.scientificbulletin.upb.ro/rev docs arhiva/full80914.pdf, (Access Date 12/02/2011) 
(7) Mario Motz, Vertical Hall sensor circuit comprising stress compensation circuit, US20140009221 (A1),

https://www.google.si/patents/US20140009221 (Access Date 01/09/2014) 